\title{
ÉVOLUTION DES FERRITES DOUX POUR TÉLÉCOMMUNICATIONS ET TÉLÉVISION
}

\author{
R. SIBILLE \\ Directeur Technique de L. C. C. Division Ferrites (COFELEC) \\ Thomson C. S. F., 74 Route de Savigny, 21204 Beaune, France
}

\begin{abstract}
Résumé. - Bien qu'il s'agisse de matériaux déjà anciens (leur développement remonte à la deuxième guerre mondiale), les ferrites doux sont encore en pleine évolution et nombreux sont ceux qui leur prédisent encore un développement industriel intense pendant encore au moins la nouvelle décennie.

Après avoir rappelé les principales caractéristiques réclamées aux ferrites pour télécommunications et pour télévision, l'auteur retrace les principales étapes de leur développement au cours des quinze dernières années : améliorations des compositions des matériaux, des circuits magnétiques, des technologies de fabrication, etc...

Les résultats obtenus sont indiqués ainsi que l'orientation actuelle des études.
\end{abstract}

\begin{abstract}
In spite of the fact that the soft ferrites are old materials (their development has been made in the 1940's), they are still in evolution and a great development in this field is predicted for the next ten years at least.

After a recall about the characteristics needed by telecommunications and television, the author reviews the main stages of the industrial development of the soft ferrites during the 15 last years : improvement of composition, magnetic circuits, technologies, a. s. o. Results are given and the trends of actual research are pointed out.
\end{abstract}

1. Généralités. - Toute industrie complexe connaît une phase de développement pendant laquelle le progrès technique est contrôlé par les caractéristiques de quelques matériaux.

Il en est ainsi pour des alliages spéciaux en Aéronautique ou pour des turbines, des combustibles pour réacteurs, du verre en Optique, etc...

Or, les ferrites sont au nombre des matières premières-clés de l'Electronique.

Variétés essentielles de matériaux magnétiques, ces céramiques dites "noires" sont des composés à base d'oxydes ou de sels minéraux qui servent à préparer des pièces détachées aux caractéristiques très diverses, de plus en plus demandées par l'industrie électronique, tant pour les matériels produits en grande série à l'usage du grand public (récepteurs radio, téléviseurs, etc...) que pour les équipements professionnels (téléphonie sur fil, télécommunications hertziennes, radar, servo-mécanismes, instruments de mesures, calculateurs, etc...) où se posent à la fois des problèmes de haute qualité sous faible encombrement et faible dérive sous l'effet de variations importantes de température et en fonction du temps.

Ils présentent sur leurs concurrents métalliques l'avantage d'avoir une résistivité considérablement plus élevée, ce qui permet d'utiliser les caractéristiques intrinsèques du matériau massif (sans qu'il soit utile de les laminer ou de les réduire en poudre).

D'autre part, comme il est possible de modifier leur composition à volonté ainsi que leur technologie de fabrication, il en existe une infinité de sortes possédant une extrême diversité de caractéristiques adaptables à chaque utilisation et pouvant conserver leurs propriétés remarquables (et en particulier leur perméabilité) dans une très large gamme de fréquences laquelle s'étend actuellement, suivant les nuances, entre quelques $\mathrm{kHz}$ et plusieurs centaines de $\mathrm{MHz}$.

Chaque domaine d'application requiert d'ailleurs des propriétés particulières, reproductibles avec une très grande précision et la plupart n'emploie que très peu de matière, eu égard à l'effet obtenu, mais par contre impose des méthodes de fabrication délicates à mettre au point et toujours assorties de contrôles fréquents et minutieux, tout au long de la chaîne, qui viennent grever considérablement le prix de revient.

L'industrie des ferrites exige donc un effort technique considérable et ne connaît cependant qu'un volume d'affaires assez limité, d'autant moins attrayant pour l'industriel que la production est morcelée en un grand nombre de sous-spécialités. Mais la qualité des produits proposés aux électroniciens contrôle à une échelle beaucoup plus vaste la qualité des équipements.

2. Historique. - Depuis les temps les plus reculés, la pierre d'aimant était connue ; il s'agissait en fait de la magnétite (oxyde magnétique noir: $\mathrm{Fe}_{3} \mathrm{O}_{4}$ ) qui est le ferrite naturel dont les qualités sont assez 
médiocres (faible perméabilité et résistivité environ $10^{3}$ fois celle du fer) comparées à celles de nos ferrites de $\mathrm{Mn}-\mathrm{Zn}$ actuels (perméabilités les plus courantes comprises entre 500 à 10000 et résistivité environ $10^{7}$ à $10^{8}$ fois celle du fer) ou à celles de nos ferrites de $\mathrm{Ni}-\mathrm{Zn}$ (perméabilités 10 à 1000 et résistivité environ $10^{12}$ fois celle du fer).

Il est en général admis que les premières études systématiques sur les ferrites ont été faites en Allemagne par S. Hilpert en 1909 puis en France par H. Forestier en 1928 dans le but de déterminer les relations existant entre les propriétés magnétiques et les compositions chimiques ; mais ces études n'ont pas abouti à des développements industriels, d'une part à cause de l'instabilité de ces premiers matériaux et également du fait d'un manque de marché potentiel : les besoins en hautes fréquences ne se faisaient pas encore sentir, aussi ces pionniers étaient-ils trop en avance sur leur temps...

Au Japon le Professeur Y. Kato et son Assistant le Professeur Takeshi Takei, tous deux de l'Institut de Technologie de Tokyo, ont étudié les ferrites dès 1930. Mais ce n'est seulement que quelques années avant la dernière guerre mondiale qu'ont commencé à être développés les premiers ferrites, d'une part au Japon, où une Société (ancêtre de TDK) a été fondée en 1935 pour fabriquer les matériaux mis au point à l'Institut de Tokyo, puis en Hollande par J. L. Snoek (à la Société Philips).

Et il a fallu attendre 1948 pour avoir une explication théorique claire du comportement des ferrites grâce au mémorable traité sur le ferrimagnétisme [1] du Professeur Louis Néel qui permit ainsi à la France de jouer un rôle déterminant dans la construction de cet édifice et la connaissance des phénomènes.

3. Ferrites pour télécommunications. - L'industrie des Télécommunications et plus généralement de l'électronique réclame toujours des pièces détachées de plus en plus petites, capables de fonctionner avec une sécurité tous les jours accrue dans une gamme de température continuellement élargie et un domaine de fréquence de plus en plus étendu.

Or, parmi toutes ces pièces détachées les inductances et les transformateurs, qui constituent les éléments essentiels de tout organe de transmission, doivent évidemment posséder de très hautes qualités et une excellente fiabilité lesquelles sont absolument indispensables pour assurer la sécurité de fonctionnement de l'ensemble ce qui pose des problèmes techniques et technologiques souvent très difficiles à résoudre, d'une part en ce qui concerne les matériaux et d'autre part l'ensemble des pièces (pots, systèmes de réglage, habillages...).

Ce haut niveau de qualité des pots pour Télécommunications est assuré grâce aux nombreux contrôles réalisés sur les matières premières et aux différents stades de la fabrication et également à l'ensemble des contrôles effectués sur les produits finis par le
Service Qualité lequel est à L. C. C. placé sous le Contrôle du Service National de la Qualité (S. N. Q.) et garantit ainsi pour chaque lot des performances conformes aux normes françaises (Qualité N. F.).

3.1 FerRITES DE Mn-Zn. - La place très importante prise par ces matériaux dans la fabrication des noyaux magnétiques pour transformateurs et pour bobines d'inductance dans la gamme des basses et moyennes fréquences (jusqu'aux environs de 1,5 à $2 \mathrm{MHz}$ ) est bien connue.

Leurs caractéristiques les plus appréciées sont leur haute perméabilité et basses pertes, combinées avec une bonne stabilité à la fois en fonction de la température, du temps (phénomène de désaccommodation $\mathrm{DF}$ ) et des chocs (thermiques, magnétiques, mécaniques...).

Leur formule chimique est : $\mathrm{Me}^{2+}, \mathrm{Fe}_{2}^{3+} \mathrm{O}_{4}$ où $\mathrm{Me}^{2+}$ désigne le plus généralement du $\mathrm{Mn}^{2+}, \mathrm{du} \mathrm{Zn}^{2+}$ et du $\mathrm{Fe}^{2+}$ et quelquefois $\mathrm{du} \mathrm{Mg}^{2+}$, du $\mathrm{Cu}^{2+}$, etc...

Les compositions les plus courantes sont donc représentées par les formules suivantes:

$$
\mathrm{Zn}_{x}^{2+}, \mathrm{Mn}_{1-x-y}^{2+}, \mathrm{Fe}_{y}^{2+}, \mathrm{Fe}_{2}^{3+} \mathrm{O}_{4},
$$

$x$ et $y$ étant fonctions des caractéristiques des ferrites à obtenir ainsi que de la technologie de fabrication. Nous rappellerons seulement le rôle très important joué par le ferrite ferreux : $\mathrm{FeO}, \mathrm{Fe}_{2} \mathrm{O}_{3}\left(\mathrm{Fe}_{3} \mathrm{O}_{4}\right)$ sur l'ensemble des caractéristiques : une légère fluctuation de la teneur en $\mathrm{Fe}^{++}$entraînant des modifications importantes de ces dernières.

Au cours des quinze dernières années, d'importantes études ont été effectuées sur ces matériaux dans le but de mieux connaître les phénomènes et les maîtriser et ainsi améliorer leurs principales caractéristiques mais parallèlement les efforts ont dû être portés sur un autre aspect du problème qui a une très grosse importance pratique : il s'agit de la reproductibilité des caractéristiques à l'échelle industrielle.

Il était en effet assez facile sur le plan laboratoire ou pour une petite production en fours dits «statiques » d'obtenir des caractéristiques de pointe mais un problème très sérieux s'est posé lorsqu'il a lallu reproduire ces caractéristiques sur le plan industriel ou de grande série c'est-à-dire lors du passage des frittages des pots dans les fours continus.

Ceci provient du fait qu'il est difficile, au cours de la montée en température, pendant le palier et le refroidissement, d'obtenir la courbe d'atmosphère optimale.

En effet, alors qu'il est nécessaire, dans la zone de montée en température ainsi que dans une partie de la zone de palier, d'avoir une atmosphère relativement riche en oxygène, il est par contre indispensable de refroidir suivant une courbe $\mathrm{p}\left(\mathrm{O}_{2}\right)=f(T)$ bien déterminée pour éviter la décomposition du ferrite, la réaction étant réversible :

$$
\mathrm{Mn}_{3} \mathrm{O}_{4}+\mathrm{ZnO}+\mathrm{Fe}_{2} \mathrm{O}_{3}+\mathrm{Q}_{\mathrm{cal}} \rightleftarrows \text { ferrite }+\mathrm{O}_{2}^{\nearrow} \text {. }
$$

Or, si l'on introduit aux différents endroits du four 
les atmosphères d' $\mathrm{O}_{2}$ ou de $\mathrm{N}_{2}$, il est difficile d'éviter, en raison des courants de convection qui s'établissent à l'intérieur du four, que les différentes atmosphères se mélangent et que l'on aboutisse à des rapports $\mathrm{O}_{2} / \mathrm{N}_{2}$ non souhaités.

Une solution consiste évidemment à travailler avec des fours continus divisés en un certain nombre de chambres séparées par des sas mais dans ce cas naissent immédiatement des problèmes difficiles de transfert de plaques parce que le four ne peut plus être en ligne droite et que, par suite, les dérivations des supports de frittage et des produits sont nécessaires. Il est cependant à noter que tous ces problèmes ont trouvé différentes solutions industrielles et que finalement ces fours ont permis de diminuer considérablement les dispersions des différentes caractéristiques des matériaux.

Les études sur les matériaux eux-mêmes ont été orientées dans deux directions correspondant aux deux principales applications de cette classe de ferrites :

- d'une part, en vue d'une augmentation de la perméabilité initiale et une diminution des pertes hystérétiques des matériaux destinés aux transformateurs à large bande et aux transformateurs d'impulsions ;

- d'autre part en vue d'une diminution du facteur de pertes tg $\delta / \mu$ (pertes résiduelles et par courants de Foucault), une augmentation de la stabilité en fonction de la température et du temps des matériaux destinés aux bobines d'inductance de haute qualité.

3.1.1 Ferrites pour noyaux magnétiques de transformateurs. - Les efforts ont porté :

3.1.1.1 Sur l'amélioration de la perméabilité initiale et la diminution des pertes hystérétiques. - On sait que la perméabilité initiale est proportionnelle au rapport $\mathrm{Js}^{2} / K$, Js étant l'intensité d'aimantation à saturation et $K$ un coefficient correspondant à l'ensemble des anisotropies (magnétocristallines, magnétostrictives et de forme).

Is dépend surtout de la composition et du cycle de frittage (densité et teneur en ferrite ferreux : $\mathrm{Fe}_{3} \mathrm{O}_{4}$ ) ; mais en fait on ne peut guère agir sur Js car du point de vue théorique Is est très limité (théorie du ferrimagnétisme) et sur le plan pratique il faut conserver au matériau un point de Curie relativement élevé (par exemple au moins $120^{\circ}$ ), ce qui restreint considérablement le choix des compositions.

Il est maintenant bien établi que pour obtenir une perméabilité élevée il faut que les conditions suivantes soient remplies :

a) Une composition initiale voisine de (en mol. \%) :

$$
52 \mathrm{Fe}_{2} \mathrm{O}_{3}, 26 \mathrm{MnO}, 22 \mathrm{ZnO} \text {. }
$$

b) Un choix judicieux des matières premières lesquelles doivent être très pures, très réactives et compatibles. c) Une structure cristalline bien définie.

En particulier, il est indispensable d'obtenir des cristaux de grandes dimensions : il existe une relation linéaire entre la valeur de la perméabilité et la dimension des cristaux [2]. D'autre part, ces cristaux doivent être homogènes et autant que possible exempts de pores et d'impuretés non magnétiques, les impuretés résiduelles étant localisées uniquement aux joints des grains [3].

Ces conditions sont malheureusement contradictoires car le diamètre des grains croît avec la température mais une température de frittage trop élevée favorise l'inclusion des pores et impuretés, lesquels affectent le mouvement des parois et créent des tensions internes (anisotropie magnétique).

Il est également indispensable d'éviter les tensions provoquées par des contractions thermiques non isotropes ou des hétérogénéités de la composition ou de la structure (en particulier à la surface des pièces).

d) Un traitement thermique optimum en atmosphère et en température. En pratique, on s'efforce d'ajuster le maximum secondaire de la courbe $\mu \mathrm{i}(T)$, dû à l'annulation de la constante d'anisotropie magnétocristalline (par compensation avec celle du ferrite ferreux), au voisinage de l'ambiante pour profiter de cette haute perméabilité dans la gamme d'utilisation.

Une teneur appropriée en ferrite ferreux $\left(\mathrm{Fe}_{3} \mathrm{O}_{4}\right)$ ayant une grande magnétostriction à saturation positive, permet également de compenser la faible magnétostriction négative du ferrite mixte de $\mathrm{Mn}-\mathrm{Zn}$. On devra aussi essayer de densifier le matériau au maximum tout en évitant des températures trop élevées qui favorisent les inclusions des impuretés et le départ du zinc (surtout si l'atmosphère est réductrice) aussi des compromis ont été trouvés avec des cycles très longs, en partie sous oxygène [4].

3.1.1.2 Sur le développement de nouvelles formes de pots. - De nouveaux modèles de petits noyaux beaucoup mieux adaptés à la technique des circuits imprimés ont fait leur apparition : les pots carrés RM.

Leur forme permet en effet un gain de surface notable (le diamètre extérieur du bobinage est identique au côté du carré circonscrit au noyau).

D'autre part, la carcasse possède des broches incorporées qui permettent :

- d'une part, la soudure des fils immédiatement après le bobinage sur la machine à bobiner elle-même, ce qui économise un temps appréciable et évite des erreurs surtout sur les transformateurs comportant de nombreuses prises,

- d'autre part, l'enfichage direct de l'ensemble du pot sur le circuit imprimé.

Le montage se trouve extrêmement simplifié du fait de l'utilisation d'un nombre minimum de pièces : deux brides-ressorts qui s'agrafent dans les encoches du circuit et permettent de maintenir sous pression les deux noyaux. En particulier le blindage et l'embase 
sont supprimés d'où un gain supplémentaire très important de place, surtout en hauteur.

Ces nouveaux circuits, plus faciles d'emploi que les pots ronds classiques, conduisent donc à une réduction importante du volume et du prix des transformateurs.

3.1.1.3 Sur l'amélioration de la rectification des pots. - Des progrès très importants ont été faits sur la rectification des pièces, permettant de diminuer l'entrefer résiduel et par suite d'augmenter les perméabilités effectives des circuits. Cette amélioration est d'ailleurs d'autant plus spectaculaire que la perméabilité initiale du ferrite est plus élevée : par exemple si on réalise un entrefer total de $1 \mu$ dans un circuit magnétique fermé de perméabilité $\mu \mathrm{i}=7500$ et de longueur effective le $\simeq 2,1 \mathrm{~cm}$ (équivalent au noyau RM 5) la perméabilité devient $\mu \mathrm{e} \simeq 5500$ soit une chute de $26,7 \%$.

Ainsi actuellement il est possible d'obtenir des inductances spécifiques $A L \simeq 5000 \mathrm{nH}$ avec des noyaux RM 5 lesquels occupent seulement une surface de $5 \times 5$ pas sur le circuit imprimé.

Toutes ces études ont ainsi conduit, en une douzaine d'années, à des améliorations substantielles des caractéristiques : la perméabilité initiale des matériaux utilisés en pots est passée d'environ 2500 à 7500 alors que les pertes hystérétiques ont été réduites approximativement dans le même rapport $\left(\eta_{\mathrm{B}} \times 10^{3}\right.$ est passé de $1,1$ à 0,35$)$.

Aussi les performances qui étaient obtenues avec un pot $22 \times 13$ de l'époque sont maintenant possibles avec un noyau RM 5 dont le volume est huit fois plus faible ( $\frac{1}{4}$ en surface et $\frac{1}{2}$ en hauteur).

3.1.2 Ferrites pour noyaux magnétiques de bobines d'inductances pour filtres. - Une des causes principales de variations de la fréquence de résonance des filtres utilisés en électronique est la variabilité de la perméabilité initiale des noyaux en ferrite qu'elles contiennent, variabilité qui est un obstacle important à l'augmentation de la perméabilité effective des circuits (la variabilité étant proportionnelle à $\mu$ e) et par suite à la miniaturisation des inductances.

Or, cette variation peut être due :

- d'une part, à des phénomènes réversibles : coefficient de température,

- d'autre part, à des phénomènes irréversibles dont le plus important, pour cette classe de matériaux, est la désaccommodation de sa perméabilité (variation en fonction du temps).

Ainsi on montre aisément que si deux noyaux géométriquement semblables sont fabriqués avec deux matériaux de perméabilités effectives $\mu_{1}$ et $\mu_{2}$ et s'ils possèdent la même variabilité, on a la relation :

$$
\frac{\mu_{2}}{\mu_{1}}=\alpha^{2}\left(\alpha=\frac{\varphi_{1}}{\varphi_{2}} \text { est leur rapport de similitude }\right) .
$$

Par exemple, si deux pots sont fabriqués avec deux matériaux (I et II) ayant les caractéristiques suivantes :

$$
\begin{array}{ccc} 
& \text { Matériau I } & \text { Matériau II } \\
\mathrm{DF} \times 10^{6} & \overline{5} & \overline{3} \\
\mathrm{TF} \times 10^{6} & 1 \pm 0,5 & 0,6 \pm 0,3
\end{array}
$$

on peut, pour obtenir la même variabilité, prendre $\mu_{2} \simeq 1,6 \mu_{1}$ et par suite $\alpha \simeq 1,25$.

Le matériau II permet ainsi de gagner une dimension dans l'échelle normalisée c'est-à-dire par exemple qu'un pot de $11 \times 7 \mathrm{~mm}$ fabriqué avec le matériau II est équivalent à un pot de $14 \times 8 \mathrm{~mm}$ fabriqué avec le matériau I (ou un $22 \times 13$ est équivalent à un $26 \times 16$ ) en ce qui concerne l'inductance, la désaccommodation et le coefficient de température, ce qui montre le rôle primordial joué par la stabilité des matériaux dans la miniaturisation des pièces.

En réalité, il faut également réduire le facteur de pertes tg $\delta / \mu$ dans le même rapport (mais ici l'effet sur la surtension sera atténué par l'influence du bobinage) et dans un rapport plus élevé les pertes hystérétiques (celles-ci étant proportionnelles au carré du $\mu$ e).

Or, des travaux très importants ont été faitsil y a une dizaine d'années pour améliorer les stabilités (T.F. et $D$. F.) et diminuer les pertes de cette classe de matériaux utilisables jusqu'aux environs de $500 \mathrm{kHz}$.

En particulier leurs coefficients de température ont été adaptés à ceux des condensateurs qui leur sont habituellement associés pour la réalisation de filtres d'où les deux classes de matériaux.

1) A coefficients voisins de zéro ( $8 \mathrm{~A}$ ou $8 \mathrm{~B}$ des normes françaises NFC 93-324) pour l'utilisation avec les condensateurs au mica ou certains condensateurs céramiques.

2) A coefficients positifs ( $8 \mathrm{C}$ ou $8 \mathrm{D}$ ) pour l'utilisation avec les condensateurs au polystyrène.

D'excellentes performances ont ainsi été obtenues avec ces matériaux grâce :

a) A un choix judicieux de la composition de base (teneur en $\mathrm{Fe}_{2} \mathrm{O}_{3}$ au voisinage de $53 \%$ en mol.) et un cycle de frittage bien adapté et parfaitement reproductible.

En effet, le facteur de température est étroitement lié à la position du maximum secondaire de la courbe $\mu \mathrm{i}(T)$ lequel dépend de la composition et de la teneur en $\mathrm{Fe}^{++}$: en particulier une différence de $0,02 \%$ en mol. sur la composition entraîne un déplacement de $1{ }^{\circ} \mathrm{C}$ de la position de ce maximum [5].

b) A des produits d'addition destinés à augmenter la résistivité du matériau et par suite à diminuer les pertes par courants de Foucault. Un des plus utilisés est le calcium [6] lequel a en général tendance à se placer autour des grains et ainsi à former une barrière isolante. D'autre part, il permet d'obtenir une porosité ouverte qui favorise la diffusion de l'oxygène vers le cœur de l'échantillon et par suite il conduit à une diminution 
de la teneur en $\mathrm{Fe}^{++}$aux joints des cristaux d'où une augmentation également de la résistivité des parois.

Mais la diminution des pertes se fait sentir surtout vers les basses fréquences car le calcium modifie peu les propriétés intrinsèques des cristaux.

Cependant, son effet peut être dopé par la présence d'une quantité déterminée de $\mathrm{SiO}_{2}$ [7], de $\mathrm{B}_{2} \mathrm{O}_{3}$ ou $\mathrm{ZrO}_{2}$ [8]. De même un effet très bénéfique sur le facteur de pertes $(\operatorname{tg} \delta / \mu)$ est obtenu par la combinaison de $\mathrm{CaO}+\mathrm{Ta}_{2} \mathrm{O}_{5}$ ou $\mathrm{CaO}+\mathrm{Nb}_{2} \mathrm{O}_{5}$ [9] ainsi que par une combinaison d'oxydes de vanadium, de lanthane, d'antimoine et de strontium : par un frittage bien adapté, la résistivité peut être multipliée par un facteur supérieur à 10 , avec seulement une concentration d'environ $0,025 \%$ de chacun des constituants [10].

c) A une amélioration des frittages (paramètre de fabrication le plus important) en fours continus qui a permis une bonne stabilisation de la teneur en $\mathrm{Fe}^{++}$ dans la composition finale et par suite une bonne régularité de toutes les caractéristiques.

Il y a quelques années d'autres études ont été entreprises dans le but de substituer des ions $\mathrm{Fe}^{3+}$ par des ions tétravalents $\left(\mathrm{Me}^{4+}\right)$ comme par exemple $\mathrm{Sn}^{4+}$, $\mathrm{Ti}^{4+}$ [11], etc... La valeur d'une quantité égale d'ions de fer se trouve alors diminuée d'une unité de façon à former un ensemble de deux ions ayant une valence moyenne de trois $\left(2 \mathrm{Fe}^{3+} \sim \mathrm{Fe}^{2+}+\mathrm{Me}^{4+}\right)$. Ainsi on obtient à partir d'un ferrite de $\mathrm{Mn}-\mathrm{Zn}$ :

$$
\begin{gathered}
\mathrm{Zn}_{x}^{2+}, \mathrm{Mn}_{1-x-y}^{2+}, \mathrm{Fe}_{y}^{2+}, \\
{\left[\mathrm{Me}_{z}^{4+}, \mathrm{Fe}_{z}^{2+}\right], \mathrm{Fe}_{2-2 z}^{3+} \mathrm{O}_{4} .}
\end{gathered}
$$

Les buts ainsi poursuivis sont multiples.

- Tout d'abord cette substitution entraîne une réduction importante de la teneur en $\mathrm{Fe}_{2} \mathrm{O}_{3}$ dans la composition de base, teneur qui est à l'origine d'une valeur élevée du D. F.

- D'autre part, en substituant un ion $\mathrm{Me}^{4+}$ à un ion $\mathrm{Fe}^{3+}$ dans la composition de base il y a apparition d'un ion $\mathrm{Fe}^{2+}$ et par suite la teneur en fer ferreux totale augmente mais, une partie équivalente de $\mathrm{Fe}^{2+}$ se trouvant liée au $\mathrm{Me}^{4+}$, les fluctuations ne portent plus en fait que sur la partie libre (y) qui peut être assez faible par un choix approprié de la composition de base.

Ainsi la présence de $\mathrm{MeO}_{2}$ permet de minimiser les variations de la teneur en $\mathrm{FeO}$ dans le ferrite final et par suite d'améliorer la reproductibilité des caractéristiques électromagnétiques.

- Enfin, la présence du $\mathrm{MeO}_{2}$ dans les ferrites de $\mathrm{Mn}-\mathrm{Zn}$ a une action sur la température de compensation qui est similaire à celle du cobalt [12] utilisé dans les ferrites de Ni-Zn, c'est-à-dire que l'augmentation de son taux déplace la courbe $\mu \mathrm{i}(T)$ vers les températures élevées. Comme l'influence du $\mathrm{Fe}^{2+}$ est inverse, on voit que l'on peut ainsi faire varier le TF dans une gamme de température déterminée et en particulier obtenir des matériaux ayant une variation presque linéaire de leur perméabilité dans une gamme assez large. Ces procédés ont également été appliqués aux matériaux hautes fréquences utilisables jusqu'aux environs de $1,5 \mathrm{MHz}$.

Dans l'ensemble les résultats obtenus sont assez remarquables, par exemple :

$$
\begin{aligned}
& \mu \mathrm{i} \simeq 2000 \quad \mu \mathrm{i} \simeq 650 \\
& \frac{\operatorname{tg} \delta}{\mu} \times 10^{6} \simeq 2 \text { à } 100 \mathrm{kHz} \frac{\operatorname{tg} \delta}{\mu} \times 10^{6} \simeq 10 \text { à } 1 \mathrm{MHz} \\
& \eta_{\mathrm{B}} \times 10^{3} \simeq 0,2 \quad \eta_{\mathrm{B}} \times 10^{3} \simeq 0,5 \\
& \mathrm{DF} \times 10^{6} \simeq 2 \quad \mathrm{DF} \times 10^{6} \simeq 5 \\
& \mathrm{TF} \times 10^{6} \simeq 1\left(\text { entre }+5^{\mathrm{o}} \text { TF } \times 10^{6} \simeq 1,5\right. \text { (entre } \\
& \text { et } \left.\left.+55^{\circ}\right)+5^{\circ} \text { et }+55^{\circ}\right)
\end{aligned}
$$

L'évolution actuelle se poursuit :

- D'une part, vers la diminution de la valeur moyenne du facteur de température des matériaux $\left(\mathrm{TF} \times 10^{6} \simeq 0,6\right)$ de façon à permettre une augmentation de la perméabilité effective et par suite une miniaturisation des circuits (comme indiqué ci-dessus).

Le gain d'une dimension de pots dans l'échelle normalisée sera ainsi possible : par exemple passage du modèle RM 6 au RM 5.

Cette miniaturisation nous oblige d'ailleurs à un affinage des cycles de frittage surtout pendant le refroidissement. En effet les échanges thermiques et surtout gazeux au cours du frittage de ces pièces sont très dépendantes de l'épaisseur des parois.

- D'autre part, vers l'extension des gammes de températures d'utilisation aussi bien vers les basses températures que vers les températures élevées : le but est d'obtenir un coefficient de température linéaire entre -40 et $+85^{\circ} \mathrm{C}$, de façon à pouvoir réaliser des inductances compatibles (dimensionnellement et électriquement) avec les condensateurs miniatures à haute stabilité, pour la fabrication des filtres à haute fiabilité exigés dans de nombreux domaines de l'électronique professionnelle (télécommunications, instruments de mesures, servomécanismes, appareils de guidage et de navigation...).

- Parallèlement, de nouvelles technologies de fabrication des poudres sont en cours d'expérimentation dans différents pays par exemple coprécipitation d'hydroxydes, cogrillage de sels, lyophilisation (sublimation sous vide), etc...

En général, les avantages espérés de ces méthodes sont les suivants :

1) une composition chimique très précise et homogène et par suite une dispersion des caractéristiques beaucoup plus réduite,

2) une meilleure réactivité : particules très fines et plus homogènes,

3) un frittage à plus basse température, etc... 
4) une suppression du préfrittage et du broyage lesquels sont à l'origine de fluctuations très importantes des caractéristiques.

3.2 Ferrites de Ni-Zn. - Les études faites il y a une quinzaine d'années sur les différentes classes de ces ferrites hautes fréquences (supérieures à $500 \mathrm{kHz}$ ) avaient permis de développer des matériaux dits de type "Perminvar » contenant une teneur très importante en $\mathrm{Fe}_{2} \mathrm{O}_{3}(58$ à $60 \mathrm{~mol} . \%$ ) et de faibles quantités de $\mathrm{CoO}$ (environ 0,5 à $1 \%$ en poids) destinées à augmenter leur résistivité et compenser leur anisotropie cristalline (réglage du coefficient de température par déplacement de la position du maximum secondaire de la courbe $\mu \mathrm{i}(T)$.

Ces ferrites possèdent de très faibles pertes et un excellent coefficient de température dans une très large gamme mais en général ce dernier n'est pas linéaire et par suite ne peut être compensé par des condensateurs. Un autre inconvénient est celui qui concerne la variation irréversible de leur perméabilité causée par des perturbations extérieures et qui vient se superposer aux phénomènes réversibles.

Parmi ces perturbations, la plus importante est en général causée par l'application d'un champ extérieur continu ou alternatif.

En effet, ces matériaux hautes fréquences présentent des cycles d'hystérésis étranglés (dits à taille de guêpe).

Lorsqu'ils sont soumis à un champ alternatif dont le niveau dépasse un certain seuil, ils perdent cette particularité et cela de façon irréversible. Il en résulte alors une augmentation très nette des pertes et de la perméabilité initiale et on ne peut alors retrouver les conditions d'origine qu'après un traitement thermique approprié (recuit à une température supérieure au point de Curie). Ces matériaux "Perminvar» présentent donc une instabilité importante qui peut être catastrophique pour le fonctionnement des filtres.

En effet, si par suite d'une cause accidentelle, la bobine est traversée par un courant continu ou alternatif important, la valeur de son inductance, entre autres, sera profondément modifiée ainsi que la courbe de réponse du filtre et ce désaccord persiste même après la suppression du défaut. Il en est de même si le pot se trouve placé accidentellement dans un champ magnétique intense ou s'il reçoit un choc.

Or, ce phénomène est général pour ces ferrites hautes fréquences à tel point qu'une méthode spéciale de préconditionnement a dû être mise au point pour permettre la mesure de la désaccommodation de ces matériaux : voir recommandation du Comité Electrotechnique International (C. E. I.).

En effet, la méthode électrique classique de préconditionnement par décharge d'un condensateur et qui est utilisée pour tous les ferrites de $\mathrm{Mn}-\mathrm{Zn}$ (décharge oscillante amortie) ne peut être employée à cause de l'instabilité de ces matériaux.

A la place le C. E. I. propose une méthode thermique et, durant tout l'essai, l'échantillon doit être protégé contre les perturbations magnétiques et les efforts mécaniques.

De même, au cours de l'utilisation de ces matériaux, des consignes très sévères doivent être respectées : en particulier, il est indispensable d'éviter les chocs magnétiques ou mécaniques, d'éloigner ces ferrites des champs parasites intenses (éviter la proximité de transformateurs ou d'aimants) de supprimer toutes les contraintes (serrages avec pièces mécaniques, enrobages, etc...).

Tous ces phénomènes de variabilité, aussi bien réversibles (coefficient de température) qu'irréversibles (désaccommodation et instabilités) étaient ainsi devenus un obstacle important à la poursuite de la réduction des dimensions des noyaux en ferrite car ils constituaient la cause principale de la dérive de la fréquence de résonance des bobines de filtres hautes fréquences. Or, du fait de l'extension de la gamme des fréquences de fonctionnement des filtres haute stabilité et de la miniaturisation des matériels, il y a sur le marché un besoin urgent de matériaux très stables pouvant être utilisés jusqu'à des fréquences supérieures à $100 \mathrm{MHz}$.

Les études actuelles ont donc pour but d'arriver à fabriquer des ferrites de Ni- $\mathrm{Zn}$ ayant une stabilité comparable à celle des $\mathrm{Mn}-\mathrm{Zn}$.

Pour cela différentes solutions existent ; elles consistent principalement :

a) à mettre au point des compositions situées au voisinage de la stœchiométrie,

b) à compenser la chute de résistivité :

- d'une part, en diminuant le diamètre des cristaux par un choix des matières premières et d'une technologie appropriés [13],

- d'autre part, en utilisant des produits d'addition bien connus tels que $\mathrm{MnO}_{2}, \mathrm{CuO}, \mathrm{Al}_{2} \mathrm{O}_{3}, \mathrm{MgO}, \mathrm{V}_{2} \mathrm{O}_{5}$, $\mathrm{Cr}_{2} \mathrm{O}_{3}$, etc... D'excellents résultats ont également été obtenus sur le coefficient de température et les pertes par courants de Foucault par l'addition de petites quantités de ferroxplana [14].

Ces nouveaux matériaux permettront ainsi la fabrication de pots miniatures (plus petits que les pots $9 \times 5$ actuellement normalisés) réglables, de haute fiabilité et possédant des dimensions homogènes avec les autres composants montés sur les circuits imprimés (en particulier les condensateurs multicouches dits « cerfeuil»).

Ils seront sans aucun doute hautement appréciés dans de nombreux domaines de l'électronique professionnelle (télécommunications, câbles multivoies téléphoniques...) et pour les applications militaires (B. L. I., systèmes de télémesures, appareils de guidage et de navigation...).

3.3 Autres ÉtUdes. - Une bobine d'inductance est en fait un organe complexe, aussi ses performances ne dépendent pas uniquement de celles du noyau ferrite. Par exemple, la stabilité de l'ensemble peut être altérée 
par une modification d'un certain nombre de paramètres parmi lesquels on peut citer :

- la variation de la pression exercée sur le noyau (ressort, contraintes dues à l'imprégnant),

- la variation des caractéristiques du circuit (par exemple une modification de l'entrefer),

- la variation de la résistance du cuivre avec la température,

- le vieillissement de l'imprégnant,

- les instabilités du système de réglage, etc...

En particulier, ce dernier joue un rôle fondamental, aussi il est indispensable que ses qualités évoluent également en parallèle avec celles des noyaux ferrites pour que la fiabilité des différents éléments constitutifs du composant reste cohérente.

Ceci explique les efforts très importants faits par les différents fabricants dans ce domaine car il est évidemment inutile de posséder des pots à très hautes performances si ces derniers ne sont plus fiables lorsqu'on introduit des bâtonnets de réglages, lesquels sont d'ailleurs absolument indispensables à leur utilisation.

4. Ferrites pour télévision. - L'utilisation des ferrites a fortement contribué à améliorer les méthodes de fabrication des récepteurs de télévision en permettant de réduire les dimensions des pièces, d'en diminuer le prix et simultanément d'en améliorer la qualité. En particulier, les ferrites ont permis d'élargir l'écran dans de très fortes proportions sans perte de brillance, d'améliorer la finesse de l'image grâce à l'augmentation de la fréquence de balayage lignes et ont ainsi contribué puissamment à la mise au point du standard à haute définition (819 lignes). Par ailleurs, leur utilisation a réduit la puissance consommée et simplifié le câblage.

En contrepartie, le développement de la télévision est la cause du développement considérable des ferrites qui s'est produit ces dernières années du fait du nombre et du volume des pièces utilisées.

Or, la télévision couleur utilise encore beaucoup plus de ferrites que le noir et blanc en quantité de pièces et en poids (au moins trois fois), à cause du plus grand nombre de fonctions et de leur complexité.

En attendant le développement industriel des systèmes de balayage par thyristors ou transistors, gros consommateurs de noyaux $\mathrm{E}$ (ou $\mathrm{E}+\mathrm{I}$ ) pour transducteurs, correcteurs, commutateurs, etc... les ferrites sont particulièrement utilisés pour la fabrication des noyaux $\mathrm{U}$ pour transformateurs de sortie ligne et des bagues pour les blocs de déviation.

4.1 NOYAUX POUR TRANSFORMATEURS DE LIGNES. En télévision les ferrites ont joué un rôle fondamental dans la mise au point d'un élément essentiel, le transformateur de puissance lequel sert à fournir la haute tension pour le tube cathodique ainsi que la tension nécessaire à la déflexion horizontale.

Ils permettent, en effet, grâce à leur perméabilité élevées et à leurs très faibles pertes par hystérésis et courants de Foucault d'augmenter la THT, et par suite d'économiser sur le courant d'étage de sortie, ce qui est très favorable à la durée de vie du tube, tout en diminuant de façon très appréciable les dimensions du transformateur et cela malgré une augmentation de l'énergie nécessaire à la déviation par suite de l'augmentation de l'angle de déviation du tube image. Or, cette énergie a encore augmenté considérablement avec la télévision couleur, le balayage des trois faisceaux réclamant une puissance beaucoup plus grande que celui de l'unique faisceau noir et blanc.

Or, par suite de ses rôles multiples, le transformateur de sortie du balayage de lignes représente un des éléments les plus critiques d'un téléviseur ; il doit, en effet, assurer une adaptation correcte des diverses impédances mises en jeu (rapports de transformation convenable) et ne pas modifier la forme des tensions c'est-àdire qu'il doit pouvoir passer au moins le dixième harmonique de la fréquence de balayage soit plus de $200 \mathrm{kHz}$.

Or, il faut absolument éviter d'être conduit à augmenter la section des noyaux des transformateurs à cause :

- D'une part, de l'augmentation correspondante de l'importance du bobinage (augmentation de sa résistance et par suite des pertes cuivre, des capacités parasites, etc...) de l'encombrement de l'ensemble et de son prix.

- D'autre part, de l'augmentation des pertes dans le circuit magnétique lui-même.

Toutes ces pertes provoqueraient un échauffement supplémentaire inadmissible, or, il ne faut pas oublier que ces problèmes d'échauffement, compte tenu des puissances en jeu, sont très critiques, aussi il est indispensable d'y remédier à chaque source de dissipation de chaleur. La solution à toutes ces difficultés était donc d'améliorer considérablement certaines caractéristiques magnétiques, du matériau ferrite destiné à la fabrication des U pour transformateurs THT, que l'on peut résumer ainsi :

- Augmentation de l'induction surtout à chaud, qui est passée d'une valeur minimale de $2900 \mathrm{G}$ $(0,29 \mathrm{~T})$ à $3400 \mathrm{G}(0,34 \mathrm{~T})$ à $100{ }^{\circ} \mathrm{C}$ pour un champ de $2,5 \mathrm{~A} / \mathrm{cm}$.

- Réduction des pertes totales qui sont passées ces dernières années d'une valeur moyenne de $120 \mathrm{~mW} / \mathrm{cm}^{3}$ à environ $80 \mathrm{~mW} / \mathrm{cm}^{3}$ à $25^{\circ} \mathrm{C}$ pour une induction de $2000 \mathrm{G}$ et une fréquence de $16 \mathrm{kHz}$.

D'autre part, il est absolument indispensable que le coefficient de température de ces pertes soit inférieur ou égal à zéro, c'est-à-dire :

$$
\begin{aligned}
& \frac{\partial W(f, B, T)}{\partial T} \leqslant 0 \text { entre }+25^{\circ} \text { et }+100^{\circ} \mathrm{C} \\
& \quad \text { pour } f=16 \mathrm{kHz} \text { et } \widehat{B}=2000 \mathrm{G}
\end{aligned}
$$

où $W$ est la valeur des pertes totales,
$f$ la fréquence,
$\widehat{B}$ la valeur de crête de l'induction,
$T$ la température. 
Si cette dernière condition n'est pas respectée, il peut en résulter des inconvénients graves dus au phénomène d'emballement : en effet, un coefficient de température des pertes magnétiques nettement positif conduit à une génération de chaleur de plus en plus grande au fur et à mesure que le noyau ferrite s'échauffe, et par suite rend ce dernier de plus en plus mauvais (les pertes augmentent et l'induction diminue).

Par contre, un coefficient de température négatif des pertes contribue fortement à la stabilité des caractéristiques des transformateurs par la suppression de cet effet d'emballement (compensation du coefficient de température positif des pertes cuivre).

Or, pour obtenir toutes ces performances, il est absolument indispensable que le ferrite ait :

a) une composition chimique bien définie dans le système ternaire $\mathrm{Fe}_{2} \mathrm{O}_{3}, \mathrm{MnO}, \mathrm{ZnO}$,

b) une teneur la plus élevée possible (compte tenu de sa composition de base) en ferrite ferreux de façon à avoir un $\sigma$ élevé (moment magnétique par gramme),

c) une faible porosité, pour avoir une induction élevée et un faible champ coercitif (faibles pertes hystérétiques),

d) un minimum d'impuretés et de gros cristaux homogènes,

$e$ ) un point de Curie très élevé (au voisinage de $200^{\circ}$ C) du fait des températures d'utilisation, etc...

Ceci ne peut être obtenu qu'en utilisant des matières premières très pures et très réactives et une technologie appropriée particulièrement en ce qui concerne le cycle de frittage mais il est bien évident que, compte tenu $\mathrm{du}$ poids de ces pièces (deux noyaux U 59 pèsent environ $200 \mathrm{~g}$ ) et de leur utilisation (matériel grand public) ces conditions doivent rester compatibles avec une exploitation industrielle de grande série dans de bonnes conditions économiques.

4.2 BAGUe DE DÉfleXiON. - Dans les tubes cathodiques à déviation électromagnétique, le pinceau électronique est déplacé sous l'action de champs magnétiques créés par des bobines de déflexion extérieures, enfilées sur le col du tube. Ces bobines créent un flux qui traverse le tube et se referme à l'extérieur; or, seul le flux intérieur au tube est utile pour la déviation, aussi on s'arrange pour que les bobines épousent étroitement le col du tube afin que tout le volume intérieur à ces bobines soit le volume utile. Les bagues en ferrite constituent le circuit magnétique du dispositif de déflexion : elles ont pour rôle de réduire la réluctance $\mathrm{du}$ circuit extérieur au tube et par suite d'augmenter le rendement.

Grâce à leur perméabilité élevée et leur très bonne tenue en fréquence elles ont, dès leur apparition (il y a déjà une bonne vingtaine d'années) supplanté tous les systèmes existants. Leur facilité de moulage leur permet d'ailleurs de s'adapter particulièrement bien à ce genre de réalisation et d'obtenir un profil conforme au cône du tube.

$\mathrm{Au}$ point de vue électromagnétique de nombreuses études ont été faites afin de déterminer le matériau le plus adapté pour les applications dans les ensembles de déflexion. Une augmentation des pertes se traduit évidemment par une énergie récupérée plus basse et une perte sur l'amplitude de balayage; mais en fait les caractéristiques magnétiques demandées au matériau ne sont pas très critiques.

Par contre, il n'en est pas de même pour les dimensions de la bague. En effet, les tolérances dimensionnelles inhérentes au procédé de fabrication des ferrites (différences de retraits et déformations au cours du frittage) sont fréquemment la source d'une variation très nette dans les performances : une modification de leur géométrie entraînant une distorsion du flux et par suite de l'image. Or, les problèmes posés par la télévision couleur sont encore considérablement plus complexes du fait que la forme tulipe de la bague est nettement plus accentuée et le profil plus critique et que le volume des pièces est nettement plus important (environ 3 à 4 fois).

Ces problèmes sont de plusieurs ordres :

a) Céramiques et dimensionnels. - La technologie de fabrication (pressage, frittage...) est très délicate du fait de la forme et du poids de ces pièces. D'autre part, la convergence des trois faisceaux électroniques pose des problèmes de précision que ne connaissait pas le récepteur noir et blanc et qui conduisent à une précision géométrique de l'ensemble qui n'est plus compatible avec la fabrication classique des pièces en céramique d'où la nécessité d'effectuer un certain nombre de rectifications très délicates qui augmentent nettement le prix de revient.

b) De matériaux. - L'accélération de la vitesse de bobinage obtenue sur les machines modernes à grande cadence ainsi que la tension importante du fil exigent des matériaux à haute résistivité de façon qu'une rupture accidentelle de l'isolement du conducteur (soit au cours du bobinage, soit au cours de l'assemblage) ne soit pas une panne pour le bloc de déflexion. Cette haute résistivité, même de l'intérieur de la masse $\mathrm{du}$ ferrite, est particulièrement exigée pour les nouvelles bagues destinées au tube $110^{\circ}$ couleur à petit col de $29 \mathrm{~mm}$ ou aux tubes PIL $90^{\circ}$ (le bobinage prévu étant toroïdal et les bagues rectifiées).

Une solution consiste évidemment à recouvrir la surface de la bague par un vernis isolant mais cette opération est en général très onéreuse aussi la solution qui a été adoptée a été le développement de matériaux à haute résistivité, par exemple des ferrites mixtes de $\mathrm{Mg}-\mathrm{Zn}$ ou de $\mathrm{Mn}-\mathrm{Mg}-\mathrm{Zn}$ et $\mathrm{Cu}$, etc..., les ferrites de $\mathrm{Ni}-\mathrm{Zn}$ étant exclus du fait du prix du NiO.

De plus les matériaux doivent être très faiblement magnétostrictifs de façon à éviter les vibrations audibles qui pourraient apparaître du fait des inductions de travail relativement importantes. 
c) De prix de revient. - Ce problème est surtout crucial pour les bagues de télévision couleur qui sont des pièces pondéreuses (environ $500 \mathrm{~g}$ ) dans lesquelles le prix des matières premières et de l'énergie intervient pour une part importante dans le prix de revient global.

Or, il est bien connu que les prix, en particulier du $\mathrm{Zn}$, $\mathrm{du} \mathrm{Cu}, \mathrm{du} \mathrm{Mn}, \mathrm{du} \mathrm{Fe} . .$. ont subi des hausses considérables l'année dernière (les prix de certains oxydes ont plus que doublé au cours du deuxième semestre 1973). Malgré cela les prix de vente de ces bagues restent assez bas en Europe du fait de l'action concurrentielle des pays de l'Est et du Japon.

d) De matières premières. - En plus de l'augmentation des prix, il existe également des problèmes d'approvisionnement pour certaines matières premières et en particulier pour les oxydes de manganèse, la plupart des gros fabricants européens (français, italiens...) ayant abandonné la fabrication de ces produits.

Ces différents paramètres (économiques et de pénurie de matières) influencent ainsi considérablement l'évolution de la composition des matériaux et par voie de conséquence la technologie de fabrication et posent en permanence des problèmes très sérieux compte tenu des quantités importantes de matières traitées.
5. Conclusions. - Les nombreux avantages des ferrites leur ont ouvert de vastes domaines d'applications qui ne cessent d'ailleurs de s'étendre.

Or, bien qu'il s'agisse de matériaux déjà anciens (leur développement remonte à la deuxième guerre mondiale) les ferrites ont subi des améliorations considérables ces quinze dernières années et ils sont encore actuellement en pleine évolution aussi nombreux sont ceux qui leur prédisent encore un développement industriel intense pendant encore au moins la prochaine décennie malgré des attaques " tous azimuts".

L'avenir à plus long terme semble par contre plus incertain et dépend du développement industriel d'un certain nombre de produits comme les filtres mécaniques ou à quartz, les photodiodes ou les cristaux liquides, etc... Il dépend également de l'évolution future de leurs performances mais d'autres améliorations ne seront possibles qu'au prix d'efforts techniques et technologiques très importants pouvant nous entraîner jusqu'à une refonte complète du processus de fabrication dans le but d'augmenter encore nettement leurs qualités et diminuer les dispersions de leurs caractéristiques, afin de permettre encore de nouvelles miniaturisations des circuits.

\section{Bibliographie}

[1] NÉEL, L., Ferrimagnétisme et Antiferromagnétisme. Annls de Phys. 3 (1948) 198.

[2] Roess, E., Moser, E., Z. Angew Phys. 13 (1961) 247.

[3] Roess, E., Proceedings of the International Conference on Ferrites (Japan) p. 203 (1970).

[4] LesCroel, Y., Ferrites de Mn-Zn à haute perméabilité C. et T. 24e, $\mathrm{A} \mathrm{n}^{\circ} 1$ (1970).

[5] Hiraga, T., Proceedings of the International Conference on Ferrites (Japan) p. 180 (1970).

[6] Guillaud, Ch., Paulus, M. et Vautier, R., C. R. Hebd. Séan. Acad. Sci. 242 (1956) 2712-2715.

[7] Axashi, T., Proceedings of the International Conference on Ferrites (Japan) p. 183 (1970).
[8] Stijntues, W., Proceedings of the International Conference on Ferrites (Japan) p. 194 (1970).

[9] Hiraga, T., Proceedings of the International Conference on Ferrites (Japan) p. 180 (1970).

[10] Roess, E., Proceedings of the International Conference on Ferrites (Japan) p. 189 (1970).

[11] Marais, A., Merceron, Th., C. R. Hebd. Séan. Acad. Sci. 257 (1963) 1760-1763.

[12] Stijntjes, W., Proceedings of the International Conference on Ferrites (Japan) p. 191 (1970).

[13] Globus, A., Thèse Fac. Sci. Paris (1963).

[14] ARAI, T., IDo, T., Proceedings of the International Conference on Ferrites (Japan) p. 225 (1970). 